\title{
HyPOSPADIAS: LONG TERM FOLLOW-UP IN A SINGLE CENTER
}

\author{
F. Molinaro, R. Angotti, E. Bindi, M. Sica, M. Aglianò, M. Messina, F. Mariscoli \\ Division of Pediatric Surgery, Department of Medical Science, Surgery and Neuroscience, \\ University of Siena, Italy
}

\begin{abstract}
Introduction: Hypospadias is one of the most common birth defects that affect the male urogenital tract. It can present as isolated anomaly, but sometimes can arise in the context of complex disorders of sex development (DSD). These malformations are characterized by a great variety of clinical manifestations and compromise the aesthetic appearance, but also functional and psychological impact that the malformation can determine the patient. Materials and Methods: We conducted a retrospective study of patients undergoing surgery for hypospadias from March 2000 to January 2015 . The data was extrapolated from a prospective database. It was considered for each patient: demographics; type of hypospadias; surgical technique; average age for surgery; intraoperative and postoperative complications (early and late). Duckett's classification was used. Results: 343 urethroplasties were performed. 320 (93\%) were primary urethroplasties and 23 (7\%) reoperations in patients who had performed many other surgical procedures. 7 patients with megameatus were excluded. The hypospadias have been ranked according to Duckett's classification, 35 patients had associated diseases. In total were performed: $186(55 \%)$ Snodgrass, $71(21 \%)$ Duckett, 10 (3\%) augmented Duckett, 42 (13\%) Magpi, 16 (5\%) Duplay, 1 $(0,3 \%)$ Bracka, $1(0,3 \%)$ was a Bianchi'technique and $5(1,4 \%)$ were Standoli. In 4 patients $(1 \%)$ were used mixed technique. There were no intraoperative complications. The mean age at surgery was 15 months (range 12-22 months). Postoperative complications were $12 \%$. Long term follow up was done with uroflussimetrie at 3 and 6 months in those who had reached the continence and possible urethral calibrations in those who had submitted a stenosis in post-op. Conclusions: The improvement of surgical techniques, the use of optical amplification tools, the use of suture material (PDS) and the experience gained in recent years have enabled us to optimized the results. Though aware of the potential and actual complications that this type of microsurgical correction can lead to the results we have obtained are comparable to those of major international series and can be considered satisfactory, both from an aesthetic and functional.
\end{abstract}

Key words: Hypospadias; disorders of sex development; microsurgical technique.

\section{INTRODUCTION}

Hypospadias is one of the most common birth defects that affect the male urogenital tract. It can present as isolated anomaly, but sometimes can arise in the context of complex disorders of sex development (DSD) [1,2].

These malformations are characterized by a great variety of clinical manifestations and compromise the aesthetic appearance, but also functional and psychological impact that the malformation can determine the patient [3].

Despite the frequent finding of the disease and the large number of surgical techniques described, it is not yet possible to indicate which of these are the best in terms of efficiency, reduction of complications, improved appearance and functional final.

The purpose of this article is, therefore, provide a review of the cases obtained in Pediatric Surgery Clinic of Siena and compare with the literature.

\section{MATERIALS AND METHODS}

We conducted a retrospective study of patients undergoing surgery for hypospadias from March 2000 to January 2015.

The data was extrapolated from a prospective database.

It was considered for each patient: demographics; type of hypospadias; surgical technique; average age for surgery; intraoperative and postoperative complications (early and late).

The Duckett's classification was used.

Preoperative management included: preoperative ultrasound of the urinary tract; psychological consultation in patients more than six years.

The postoperative follow-up included: clinical evaluation one week after discharge, 3 months and one year; uroflowmetry after 1 year from the achievement of continence and then depending on the patient.

Correspondence to:

Mariscoli Francesca

Division of Pediatric Surgery, Dept of Medical Sciences, Surgery and Neuroscience, University of Siena, Polyclinic "Le Scotte" viale Bracci 16, 53100 Siena, Italy

Tel.: +39.577.586502; Fax: +39.577.586174

E-mail: fragne85@hotmail.it 


\section{RESULTS}

343 urethroplasties were performed. 320 (93\%) were urethroplasties primary and $23(7 \%)$ reoperations in patients who had performed many other surgical procedures. 7 patients with megameatus were excluded. The hypospadias have been ranked according to Duckett's classification (Table 1), 35 patients had associated diseases (Table 2).

In total were performed: $186(55 \%)$ were Snodgrass, $71(21 \%)$ were Duckett, $10(3 \%)$ were Augmented Duckett, 42 (13\%) were Magpi, 16 (5\%) were Duplay, 1 $(0,3 \%)$ Bracka, $1(0,3 \%)$ was a White and $5(1.4 \%)$ were Standoli. In 4 patients ( $1 \%$ ) were used mixed media (4Duckett/Nesbit and 1-Snodgrass/Duplay) (Table 3).

There were no intraoperative complications.

Table 1. Percentual of patient operated according to the Duckett's classification of the hypospadias.

\begin{tabular}{lc}
\hline Patient & N. (\%) \\
\hline Anterior & $200(58)$ \\
Glandular & $42(21)$ \\
Balanopreputial sulcus & $103(51.5)$ \\
Penile anterior & $55(27.5)$ \\
\hline Medium & $45(13)$ \\
With cordhee & $14(31)$ \\
Without cordhee & $31(69)$ \\
\hline Posterior & $68(22)$ \\
Posterior & $22(32.3)$ \\
Penoscrotal & $36(53)$ \\
Perineal & $10(5$ DSD + vagina) (14.7) \\
\hline Multisurgical & $23(7)$ \\
\hline
\end{tabular}

Table 2. Associates disease.

\begin{tabular}{lccc}
\hline Disease & N. & \multicolumn{2}{c}{ Hypospadia } \\
anterior & posterior \\
\hline Unilateral cryptorchidism & 9 & 6 & 3 \\
\hline Bilateral cryptorchidism & 7 & 4 & 3 \\
\hline Epileptic encephalopathy & 2 & - & 2 \\
\hline Anorectal malformation & 3 & 3 & \\
\hline Renal agenesis & 5 & 2 & 3 \\
\hline Vescicoureteral reflux & 3 & 1 & 2 \\
\hline Esophageal atresia & 1 & 1 & - \\
\hline Scrotum vulva's type & 2 & - & 2 \\
\hline Sketch vaginal residue & 5 & - & 5 \\
\hline
\end{tabular}

The mean age at surgery was 15 months (range 1222 months).

Postoperative complications were $12 \% .24$ patients (7\%) had one or more fistulas. Of these 13 they had had surgery for anterior hypospadias and 10 posterior (Table 4).

The average time of diagnosis/fistula/urethral and was 6 months ( 2 weeks-24 months).

In all it was performed the closure of the fistula, 6 months after surgery. 1 patient (treated in an another hospital) needed two operations and two patients of 3 interventions.

Ten patients (3\%) had a urethral stricture. The symptoms occurred in an average of 7 weeks of surgery. The 10 patients underwent preoperative uroflowmetry and showed a pattern uroflussimetric

Table 3. Surgical techniques used.

\begin{tabular}{lc}
\hline Technique & N. (\%) \\
\hline Anterior/medium without cordhee & $231(67.3)$ \\
Snodgrass & $173(50.4)$ \\
Magpi & $42(12.2)$ \\
Bianchi & $1(0.3)$ \\
Duplay & $15(4.4)$ \\
\hline Medium with cordhee/posterior & $82(23.9)$ \\
Duckett & $64(18.6)$ \\
Augmented Duckett & $9(2.6)$ \\
Duckett + Nesbitt & $3(0.9)$ \\
Bracka & $1(0.3)$ \\
Standoli & $4(1.2)$ \\
Snodgrass + Duplay & $1(0.3)$ \\
\hline Multisurgical & $23(6.7)$ \\
Snodgrass & $13(3.8)$ \\
Duplay & $1(0.3)$ \\
Duckett & $7(2)$ \\
Augmented Duckett & $1(0.3)$ \\
Standoli & $1(0.3)$ \\
\hline
\end{tabular}

Table 4. Post-surgical complications.

\begin{tabular}{lc}
\hline Complication & N. (\%) \\
\hline Fistulas & $24(6)$ \\
\hline Hypospadia anterior & $14(6)$ \\
\hline Hypospadia posterior & $10(12)$ \\
\hline Stenosis & $10(2.9)$ \\
\hline Subtotal dehiscence & $5(1.4)$ \\
\hline Urethral diverticulum & $1(0.3)$ \\
\hline
\end{tabular}


with an average Qmax $<10 \mathrm{~mL}$ (range $=8-12 \mathrm{~mL}$ ). 6 patients underwent a single calibration to a month after surgery urethroplasty, two were required with multiple calibrations and 2 of meatus plastic [2-4]. 3 of these have had a final resolution, 1 continues to present a stenosis, for which we set from about 2 months a program of home care expansion thanks to the compliance of the parents and the small with Nelaton catheters $\mathrm{Ch}$ [6-8] three times a day. All patients treated for urethral stricture finally performed a calibration uroflowmetry post about three months and then six months with normalization of urinary stream. 5 patients $(12.5 \%)$ presented a subtotal of urethroplasty dehiscence around after 10 weeks of surgery (1-24 weeks), necessitating an intervention in two stages with a flat opening of the urethra and subsequent "Redu according to Snodgrass" at a distance of at least 6-8 months after the first half.

Only one patient presented at 12 months after surgery a urethral diverticulum diagnosed with HCM and a cystoscopy, surgically removed.

\section{DISCUSSION}

Treatment of hypospadias is essentially surgical and the last 15 years has undergone major changes.

A surgical correction of hypospadias performed successfully can get proper erection of the penis, a new meatus at the top of the glans, a urination in standing and normal sexual relations.

Although it is a very common disease it is not yet clear what the best surgical technique.

Our study was retrospective which recruited 343 patients.

The study population included patients with both proximal and distal hypospadias, is subject multi operated from another hospital. According to the literature patients undergoing surgery showed a mean age of about 15 months, which is the best age for 'surgery [4]. Tekgül S et al in the Guidelines of Pediatric Urology indicates the best age for surgical treatment from 6 to 18 months.

Over the years we have been used several techniques of correction. In the literature, it is not mentioned any technique as a gold standard, for both forms anterior or posterior and the choice seems to be dictated by the experience of the single center, surgeon, rather than from the comparative data. In our department for anterior hypospadias surgery of choice is urethroplasty sec. Snodgrass, with preputial flap sec. Retik. For distal hypospadias, the technique used was the urethroplasty as the Duckett's technique.

The postoperative complications appeared in $12 \%$ of our patients, of which $6 \%$ of fistulas, stenosis of $2.9 \%$, $1.4 \%$ subtotal anastomotic leakage of the urethroplasty and $0.3 \%$ of the formation of urethral diverticulum.
Fistula in our series is the complication most represented, especially in the posterior hypospadias $(12 \%)$ than the anterior $(6 \%)$, and the diagnosis was made at 6 months after surgery ( 2 weeks- 24 months).

The patients performed a follow-up post-operative one week after discharge, three months and one year [5-6].

LQ Huang et al., presented a case study of 167 patients with hypospadias front and back underwent surgery urethroplasty appearance of the fistula $12 \%$ of posterior hypospadias and $30 \%$ of anterior hypospadias. In terms of post-operative complications in our data, compared with the more recent literature, its show excellent results comply with the relevant international case studies.

Functional assessment in children not yet continents is difficult, they may be asked questions to patients and parents to get information about urination and urinary stream, but more accurate data are obtained through the uroflowmetry, according to the parameters of the International Children's Continence Society (ICCS) [4-8]. Some studies recommend performing a uroflowmetry after the child has reached the continence and the follow-up of patients showing parameters of obstruction to urine flow or borderline values [8-11]. There are papers showing that the location of the meatus and/or the surgical technique used questionnaire predict functional outcome, rather it seems to be related to the degree of severity of the chorda and the emptying of urine. Neither the location nor the meatus the surgical technique are predictors of outcome functional. You can distinguish symptomatic patients, for example, those exhibiting poor urinary stream, incontinence, drip, hesitation during urination and patients with subclinical symptoms as dysfunction or bladder hyperreactivity, whose diagnosis is sometimes late because of the difficulty in highlighting these aspects.

In our series all patients underwent uroflowmetry one year after achieving continence and then were evaluated according to the clinical application; in particular those with stenosis were subjected to repeated uroflussimetrie in the follow-up at a distance.

\section{CONCLUSIONS}

The analysis of our study found no substantial differences from the International Literature in terms of timing of the intervention, to choose the most appropriate surgical technique, duration of follow-up and incidence of complications.

Improving operating techniques, the use of instruments of optical amplification, of suture material and the experience gained in recent years has allowed the Pediatric Surgery Clinic of Siena to optimize their results. Although we are aware of the possible complications that this type of correction may involve 
microsurgical, the results obtained by us are comparable to those of the major international case studies and can be considered satisfactory from both an aesthetic and functional point of view. The data collected in this study, according to the literature, demonstrate the importance of a correct timing of the intervention that is being pursued performing correction of the malformation at a time to allow the small patient does not remember the operation, of an adequate choice of the type of intervention between the various techniques used, a proper follow-up postoperative, aimed not only to an early recognition of complications but also to the long-term outcome evaluation in these patients. It is noted today a 'growing interest, not only for the correction of the functional defect, but also to the psychological consequences that such congenital malformation may result in the patient, in his life of relationship and in the parents.

\section{REFERENCES}

1. Macedo A Jr, Rondon A, Ortiz V. Hypospadias Curr Opin Urol 2012;22:447-52
2. Mouriquand P. Etiological aspect of hypospadias. Dial Peiatr Urol 2007;28:1

3. Schönbucher VB, Landolt MA, Gobet $R$, et al. Health-related quality of life and psicological adjustment of children and adolescent with hypospadias J Pediatr 2008;152:865-72.

4. Tekgül S, Riedmiller H, Dogan HS, et al. Guidelines on paediatric urology. Eur Assoc Urol, 2012. Available from: https://uroweb. org/wp-content/uploads/22-Paediatric-Urology_LR.pdf

5. McNamara ER, Schaeffer AJ, Logvinenko T, et al. Management of proximal hypospadias with 2-stage repair: 20- year experience $J$ Urol 2015;194:1080-5.

6. Huang LQ, Ge Z, Tian J, et al. Retrospective analysis of individual risk factors for urethrocutaneous fistula after onlay hypospadias repair in pediatric patients. Ital J Pediatr 2015;41:35.

7. Manzoni GA, El-Ghoneimi A. posterior hypospadias Dial Pediatr Urol 2008;29:1-11.

8. Gonzalez R, Ludwikowski BM. Importance of urinary flow studies after hypospadias repair: a systematic rewiew Int. J Urol 2011;18:757-61.

9. Snodgrass W, Villanueva C, Bush NC. Duration of follow-up to diagnose hypospadias urethroplasty complication J Pediatr Urol 2014;10:208-11.

10. Veveridis M, Dickson AP, Gough DCS. An objective assessment of the result of hypospadias surgery. BJU Int 2005;96:135-9.

11. Spinot AF, Poelart F, Groen LA. et al. Hypospadias repair in a single center: long term follow-up is mandatory to detect the real complication rate. J Urol 2013;189:2276-81. 